\title{
Computational Identification of Conserved microRNAs and Their Targets in Tea (Camellia sinensis)
}

\author{
Akan Das ${ }^{1}$, Tapan Kumar Mondal ${ }^{1,2 *}$ \\ ${ }^{1}$ Biotechnology Laboratory, Faculty of Horticulture, Uttar Banga Krishi Viswavidyalaya, Cooch Behar, West Bengal, India; ${ }^{2}$ National \\ Research Center of DNA Fingerprinting, National Bureau of Plant Genetic Resources, New Delhi, India. \\ Email: *mondaltk@yahoo.com
}

Received November $2^{\text {nd }}, 2010$; revised November $15^{\text {th }}, 2010$; accepted November $22^{\text {nd }}, 2010$.

\begin{abstract}
MicroRNAs (miRNAs) are a class of 22 nucleotides long non coding RNA molecules which play an important role in gene regulation at the post transcriptional level. The conserved nature of miRNAs provides the basis of new miRNA identification through homology search. In an attempt to identify new conserved miRNAs in tea, previously known plant miRNAs were used for searching their homolog in a tea Expressed Sequence Tags and full length nucleotide sequence database. The sequences showing homolog no more than four mismatches were predicted for their fold back structures and passed through a series of filtration criteria, finally led us to identify 13 conserved miRNAs in tea belonging to 9 miRNA families. A total of 37 potential target genes in Arabidopsis were identified subsequently for 7 miRNA families based on their sequence complementarity which encode transcription factors (8\%), enzymes (30\%) and transporters $(14 \%)$ as well as other proteins involved in physiological and metabolic processes (48\%). Overall, our findings will accelerate the way for further researches of miRNAs and their functions in tea.
\end{abstract}

Keywords: Camellia sinensis, Computational Identification, Expressed Sequence Tags, microRNA, Targets

\section{Introduction}

MicroRNAs (miRNAs) are short ( $22 \mathrm{nt})$, endogenous non coding RNAs that play an important role in many biological processes [1]. They are generated from long precursor molecules which can fold into hairpin seconddary structures [2]. Mature miRNAs bind to the complementary sites on target mRNAs and repress post transcriptional gene expression in both animals and plants [3, 4]. In plants, miRNAs are involved in diverse aspects of plant growth and development such as leaf morphology and polarity, root formation, transition from juvenile to adult vegetative phase and vegetative to flowering phase, flowering time, floral organ identity and reproduction $[5$, 6]. They are also found to be involved in response to pathogen invasion [7], hormone signaling [8,9], environmental stress $[10,11]$ and promotion of anti-viral defence [12].

Expressed Sequence Tags (ESTs) are complementary DNA (cDNA) sequences, usually $200-500 \mathrm{bp}$ in length that represents the expressed portions of genes. Therefore, ESTs can be used in gene identification, expression pro- filing and polymorphism analysis [13]. The EST sequencing projects have been enormously successful in the framework of many genome projects. The EST sequences are being used intensely as a source of information for the discovery of new genes whose functions can tentatively be deduced from their sequence and verified experimentally. Recently, in silico identification of miRNAs in various plant species have been done by EST analysis [14-17]. The biogenesis of miRNAs suggests that it is possible to find new miRNAs by homology searching of known miRNAs in ESTs. Hence, EST analysis makes it possible for studying conserved miRNAs and their functions in species whose genome sequences have not been well known $[10,18,19]$.

There are both experimental and computational approaches for the investigation of plant miRNAs. The computational approach has been proved to be faster, affordable and more effective. Most of the miRNAs in miRBase [20] have been contributed through computational approach only. The computational approach has been developed on the principle of looking conserved sequences between different species which can fold into 
hairpin secondary structures [10]. In recent years, a number of programs and bioinformatics tools have been developed and used successfully for the identification and analysis of miRNAs and their targets $[21,22]$.

Tea is one of the most important non-alcoholic beverage drinks worldwide and gaining further popularity as an important 'health drink'. Despite the limited genome resources of tea (Camellia sinensis), published EST and full length nucleotide sequences in GenBank (http://www. ncbi.nlm.nih.gov/genbank/) has provided the scope to get more genetic information. In this study, new miRNAs were mined in local tea sequence database for the purpose of understanding their roles in regulating growth and development, metabolism and other physiological processes in tea.

\section{Methods}

\subsection{Collection of Reference miRNAs, Full Length Nucleotides and EST Sequences}

All available plant miRNAs and their fold back sequences were obtained from miRBase (http://www.mirbase.org/) on May, 2010. The homolog miRNAs were eliminated and the rest were defined as reference for searching tea
miRNAs. Tea nucleotide and EST sequences (14819 as on May, 2010) were downloaded from NCBI's nucleotide and dbEST database (http://www.ncbi._nlm.nih.gov/). All redundant and poor quality sequences were eliminated and created a local nucleotide database.

\subsection{Potential miRNAs and Their Precursors}

The procedure for searching conserved tea miRNA homologues is summarized in Figure 1. The reference sequences were used as a query for homology search against our local tea nucleotide sequence database at e-value threshold $<0.01$ using BLAST + 2.2.22 program [23]. The target sequences with no more than four mismatches were considered for secondary structure prediction using Mfold v 3.2 [24]. The precursor sequences were searched at 50 nucleotides upstream or downstream from the location of mature miRNAs with an increament of 10 nucleotides. While selecting a RNA sequence as a candidate miRNA precursor, following criteria were used according to Zhang et al. [1] with minor modifications as: 1) a RNA sequence can fold into an appropriate stemloop hairpin secondary structure, 2) a mature miRNA sequence site in one arm of the hairpin structure, 3) miRNAs

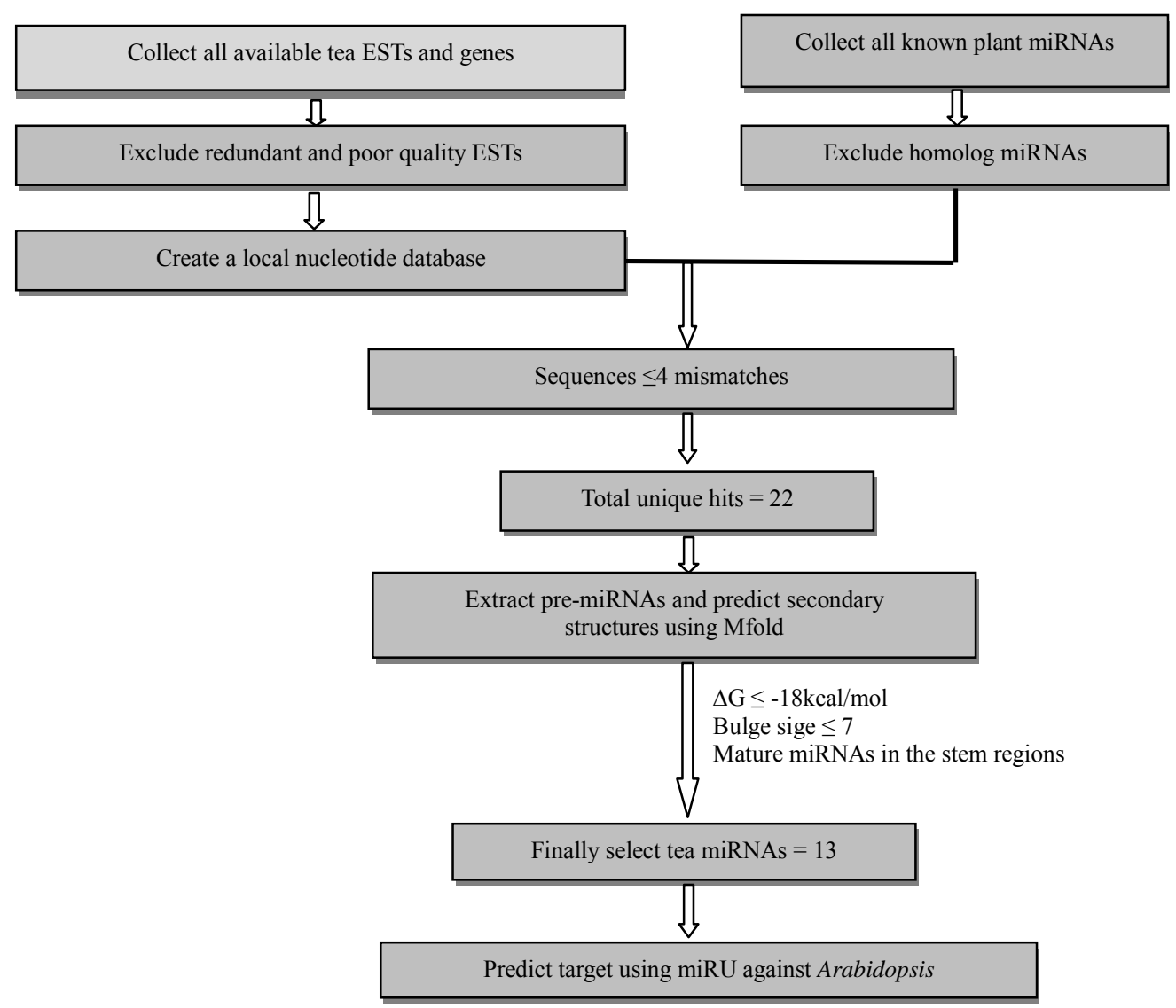

Figure 1. An overview of different steps involved in miRNA and their target prediction. 
had less than seven mismatches with the opposite miRNA sequence in the other arm, 4) no loop or break in miRNA sequences, 5) predicted secondary structures had higher negative energy MFEs ( $\leq-18 \mathrm{kcal} / \mathrm{mol}$ ), and iv) $40-70 \%$ $\mathrm{A}+\mathrm{U}$ contents.

\subsection{Prediction of Targets}

As for tea, since only few gene sequences available, we used Arabidopsis as a reference system for finding the targets of the candidate miRNAs. The predicted tea miRNAs were used as query against the Arabidopsis thaliana DFCI gene index (AGI) release 13 using miRU (http://bioinfo3.noble.org/psRNATarget/) following the criteria as 1) maximum expectation value 3 ; 2) multiplicity of target sites 2 ; 3 ) range of central mismatch for translational inhibition 9-11 nucleotide; 4) maximum mismatches at the complementary site $\leq 4$ without any gaps.

\subsection{Phylogenetic Analysis}

Due to the conserved nature of small RNAs, orthologue discovery can be done through bioinformatics analysis. We analysed tea small RNA conservation with their orthologues. A homology search of candidate tea miRNAs was done against all plant miRNAs using NCBI standalone BLAST [23] allowing maximum of 3 mismatches and e-value $<0.001$. The corresponding precursor sequences of homolog small RNA's were identified and collected (Appendix). The collected sequences of diverse plant species were aligned with homolog tea miRNA using Clustal W [25].

A query of tea small RNAs against known miRNA families (miRBase, release 15, http://www.mirbase.org/) allowed us to identify 3 previously reported large families. The precursor sequences of three known family members were selected along with respective precursor sequences of tea (Appendix). Then, the maximum likelyhood trees were constructed for each family based on Tamura-Nei model [26] with default bootstrap values using MEGA 4.0 [27] to illustrate the evolutionary relationships among the members of the family.

\subsection{Nomenclature of miRNAs}

The predicted miRNAs were named in accordance with miRBase [28]. The mature sequences are designated 'miR', and the precursor hairpins are labeled as 'mir' with the prefix 'csi' for C. sinensis, 'cas' for C. assamica and ' $c j a$ ' for $C$. japonica. In the cases where distinct precursor sequences have identical miRNAs with different mismatch pattern, they were named as csi-mir-1-a and csi-mir-1-b.

\section{Results}

\subsection{Prediction of miRNAs}

A total of 14,819 sequences containing 2,023 full length nucleotides and 12,796 ESTs were obtained from GenBank. Out of these, 22 sequences had less than five mismatches with previously known plant miRNAs. After carefully evaluating the hairpin structures using the criteria mentioned in the method, 13 small RNAs were finally identified from different species of Camellia namely sinensis, japonica and assamica. Details of the predicted miRNAs such as source sequences, location in the source sequences, length of precursor sequences and their minimum folding free energies and $\mathrm{A}+\mathrm{U}$ content are tabulated below (Table 1). A total of 9 miRNAs were

Table 1. Details of the predicted miRNAs in tea.

\begin{tabular}{|c|c|c|c|c|c|c|c|c|c|c|c|}
\hline New MiRNAs & NS & Gene ID & Strand & SP & EP & ME & Mature MiRNAs & E Value & P L & $\begin{array}{c}A+U \\
(\%)\end{array}$ & MFE \\
\hline csi-miR 408 & EST & 206583693 & 3 , & 137 & 117 & $18 / 21$ & CUGCACUGCCUCUUCCCUGAG & 0.001 & 336 & 45.24 & -120.1 \\
\hline csi-miR1171 & EST & 171355265 & 5 , & 286 & 308 & $22 / 23$ & UGGAGUGGAGUGAAGUGGAGUGG & $3 \mathrm{E}-04$ & 181 & 56.98 & -45.97 \\
\hline csi-miR414a & EST & 206583641 & 3 , & 757 & 637 & $18 / 21$ & UCUUCCUCAUCAUCAUCUUCU & 0.001 & 663 & 57.32 & -63.18 \\
\hline csi-miR414d & EST & 284026209 & 3 ' & 186 & 166 & $20 / 21$ & UCAUCGUCAUCGUCAUCAUCU & 0.004 & 193 & 61.14 & -37.72 \\
\hline csi-miR414f & EST & 212378632 & 5 , & 122 & 142 & $20 / 21$ & UCAUCAUCAUCAUCAUCUUCA & $6 \mathrm{E}-05$ & 68 & 57.35 & -18.5 \\
\hline cas-miR1122 & FL & 214011104 & 5 , & 214 & 237 & $20 / 24$ & UACUCCCUCCGUCCCAAAAUAAUG & $6 \mathrm{E}-05$ & 294 & 69.83 & -91.23 \\
\hline csi-miR414g & EST & 51453040 & 3 ' & 474 & 454 & $18 / 21$ & CCUUCCUCAUCAUCAUCGUCC & 0.001 & 70 & 45.71 & -25.2 \\
\hline csi-miRf10132-akr & EST & 51453383 & 3 , & 58 & 34 & $25 / 25$ & GCGAGCUUCUCGAAGAUGUCGUUGA & 9E-08 & 200 & 49.00 & -69.5 \\
\hline cja-miR2910 & FL & 1777723 & 5 , & 1262 & 1282 & $21 / 21$ & UAGUUGGUGGAGCGAUUUGUC & $1 \mathrm{E}-05$ & 301 & 49.83 & -91.0 \\
\hline csi-miR2914 & FL & 34787361 & 5 , & 345 & 367 & $22 / 23$ & UAUGGUGGUGACGGGUGACGGAG & $5 \mathrm{E}-06$ & 65 & 49.23 & -20.9 \\
\hline cas-miRf10185-akr & EST & 221071827 & 3 , & 232 & 212 & $17 / 21$ & GAAAGGGGAAAACAUUGUAGC & 0.004 & 139 & 48.92 & -51.1 \\
\hline cas-miR11590-akr & EST & 212379609 & 3 , & 113 & 94 & $17 / 20$ & UUUUGGUGUGCCUUCAACCU & 0.003 & 75 & 53.33 & -23.8 \\
\hline csi-miR414h & EST & 295345415 & 3 ' & 79 & 58 & $17 / 21$ & UCAUCCUCAUCAUCGUCAGAA & 0.004 & 644 & 55.36 & -86.83 \\
\hline
\end{tabular}

NS $=$ Nucleotide Source, FL = Full-Length, SP = Start Point, EP = End Point, ME = Match Extent, PL = Pre-miRNA Length, MEF = Minimal Free Energy 
predicted from ESTs whereas 4 were from full length nucleotide sequences. Five of them were located in the direct strand and the rest were in indirect strand. The newly identified precursor miRNAs have minimum folding free energies (mfe) ranging from -186.83 to $-18.5 \mathrm{kcal} / \mathrm{mol}$, with an average of about -72.69 $\mathrm{kcal} / \mathrm{mol}$ and the $\mathrm{A}+\mathrm{U}$ content were ranges from 45.24 to $69.83 \%$ with an average of $53.79 \%$. The length of the precursors ranges from 65 to $663 \mathrm{nt}$ with an average of $248 \mathrm{nt}$ and mature sequences ranges from 20 to $25 \mathrm{nt}$. The newly predicted two tea miRNA (cja-miR2910,
csi-miRf10132-akr) sequences were perfectly (100\%) matched with the corresponding homologues of populus and rice, whereas the remaining 11 mature miRNA sequences differ by 1 to 4 nucleotides from their homologues. All the mature miRNAs were found in the stem portion of the hairpin structures (Figure 2) containing less than 7 mismatches in the other arm without break or loop inside the se- quences. It was found that tea miRNA (csi-miR408) has been conserved with diverse plant species (Figure 3) from monocotyledonous plants such as rice, maize to dicotyledonous plants such as populous.

cas-miR1122

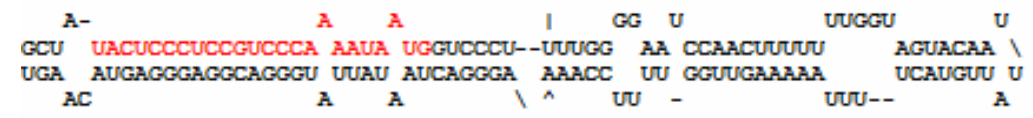

csi-miR414f

I UGC GA UU UUC- UU
CUGAG UGGUGGUG GGUG UC UUC I
GACUU ACUACUAC CUAC AG AAG C

csi-miR1171

A A AGA GU G AGG-I - - AG C

GUG GGUGA G AGGU AGGU UGU ACCUCCUU C

G A A-- UG G GAGG

cja-miR2910

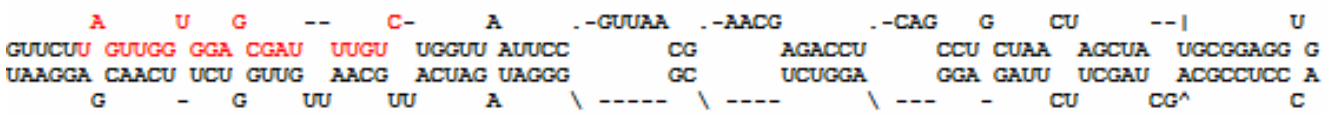

csi-miR2914

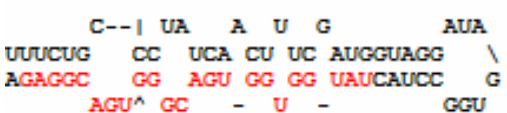

csi-miR 408

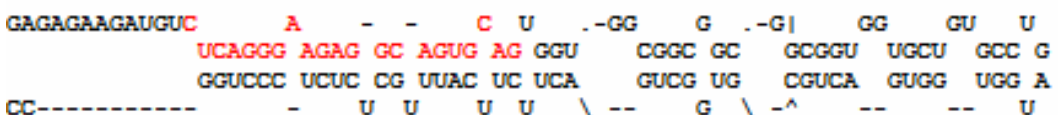

csi-miR414a

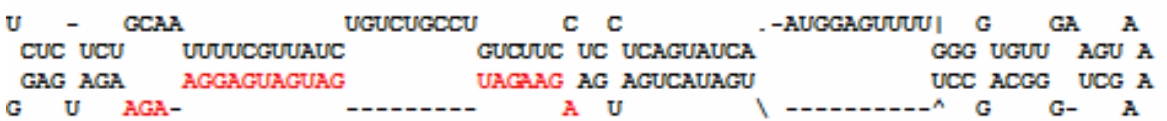


csi-miR414d

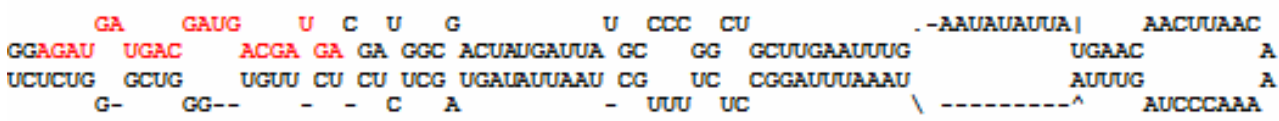

csi-miR414g

\begin{tabular}{|c|c|c|}
\hline A & -- & AGGAAGGAGALA| $\mathbf{A}$ \\
\hline GAG AGGAGGA & CGAUGAU AUG & AUC G \\
\hline CUC UCCUCCU & GCUACUG UGC & UGG G \\
\hline
\end{tabular}

csi-miRf10132-akr

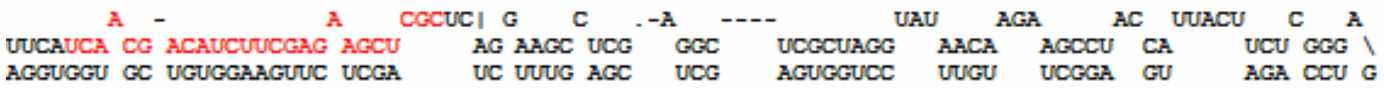

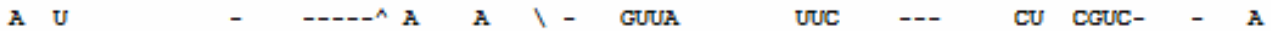

cas-miRf10185-akr

\begin{tabular}{|c|c|c|c|c|c|}
\hline \multicolumn{2}{|c|}{ UACAA } & & -1 & CCAA & GGGGAA \\
\hline CCUGC & UGUUUUCCCCUUUCUCC & GUUCUCUUUG & $\operatorname{cog} C$ & AUUUUCCGC & G $\quad 1$ \\
\hline GGCCG & ACGAAGGGGGGGAGGGG & CAGGOGAAAC & GGCG & UAAAAGUU & U \\
\hline
\end{tabular}

cas-miR11590-akr

$\begin{array}{lllll} & -1 & \text { G } & - & \text { AAAA-I AAG UU } \\ \text { GUAAGGUUG } & \text { AA GC ACACC } & \text { CA UUCA I } \\ \text { CGUUCCAAC UU CG UGUGG GU AGGU U }\end{array}$

csi-miR414h

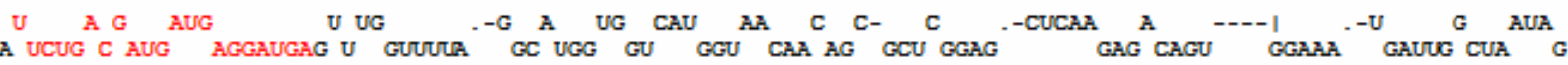

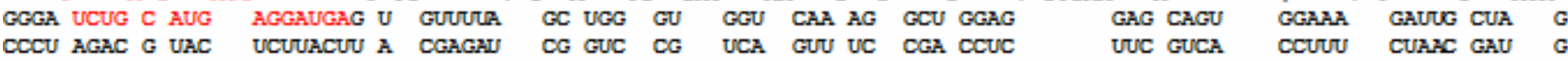

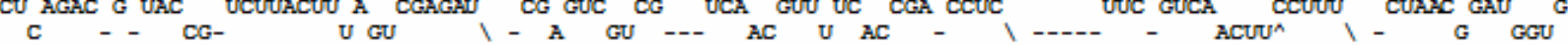

Figure 2. Predicted hairpin secondary structures of pre-miRNAs. MiRNAs are highlighted (red color) in the stem portion.

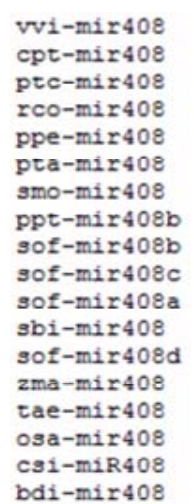

AAGCUGUUUUUGCUCUACCCAUGCACUGCCUCUUCCCUGGCUCUGUCUCUC-.-.-.-DCUCUGUUUUGGCUCCUCCCAUGCACUGCCUCUUCCCUGGCUCUCUGCCUU--:---:-UACUUGUUUUGGCUCUACCCAUGCACUGCCUCUUCCCUGGCU-UGUGGCUC--..--..CAUCUGUUUUGGCUCUACCCCUGCACUGCCUCUUCCCUGGCUUCCG-..GAGCUGUUGUGGCUCUACUCAUGCACUGCCUCUUCCCUGGCUGCCGUCCUC-:--.--CGUCUGCUUCUGCCAUUCUUAUGCACUGCCUCUUCCCUGGCUC--:--:CGGCUCCUUGUGUGGCUUUGAUGCACUGCCUCUUCCCUGGCUGCGGCCAAGUUA--:--:UGAGG----GUGUUGUCCUCAUGCACUGCCUCUUCCCUGGCUUC--CCUACAUAGCUCGC GGU-GUUGUUGCUUCCUCCCCUGCACUGCCUCUUCCCUGGCUCCCCACCGUUGCCCUUGC GGU-GUUGUUGCUUCCUCCCCUGCACUGCCUCUUCCCUGGCUCCCCACCGUUGCCCUUGC G----UUGUUGCUUCCUCCCCUGCACUGCCUCUUCCCUGGCUCCCCACCGUUGCCCUUGC GGU-GUUGUUGCUUCCUCCCCUGCACUGCCUCUUCCCUGGCU-GGU-GUUGUUGCUUCCACCCCUGCACUGCCUCUUCCCUGGCUCCCCACCGUUGCCCUUGC --U-GUUGUUGCUCCCUCCCCUGCACUGCCUCUUCCCUGGCUCCGAUCCCCCACCGUUGC --U-GUUGUUGCUCCCUCCC-UGCACUGCCUCUUCCCUGGCUCCCCUCC-CAAAUCUCUC --U-GUUGUUGCUCCCUCCCCUGCACUGCCUCUUCCCUGGCUCCCCUGCACACCUCUCUC - CUGCACUGCCUCUUCCCUGAG-ACUAGCUAGCAACAGAAUCCAUGCACUGCCUCUUCCCUGGGGAUCGAUCAUCAAAAGAGG

Figure 3. Conservation of tea miRNA (csi-miR408) with diverse plant species. Conserved portion is highlighted. Abbreviated names are given in full in appendix. 


\subsection{Phylogenetic Analysis}

The newly identified tea miRNAs belong to 9 miRNA families including three known independent large miRNA families (mir 408, mir414 and mir1122). There are one tea miRNA namely csi-miR 408 and cas-miR1122 in each family of mir-408 and mir-1122, respectively. However, five members of family mir 408 were found in tea (csi-miR414a, csi-miR414d, csi-miR414f, csi-miR414g, and csi-miR414h). The comparison of the predicted miRNA precursor sequences with other members in the same family showed that most members could be found to have a high degree of sequence similarity with others. The phylogenetic trees among the members of each family illustrated the evolutionary relationships of tea miRNAs (Figure 4).

\subsection{Target Prediction}

A total of 37 potential targets were identified for the 7 predicted miRNA families which include 11 miRNAs based on their perfect or nearly perfect complementarity with their target sequences in Arabidopsis (Table 2, Figure 5). For all the miRNAs, single binding site was found in the targets without any gaps in the complementtary region and expectation value ranges from 0 to 3 . These potential miRNA targets were belonged to a number of gene families that involved in different biological functions such as regulation of cell cycle, metal ion transportation, starch metabolic processes etc. There were $8 \%$ of genes encoding transcription factors, $30 \%$ of

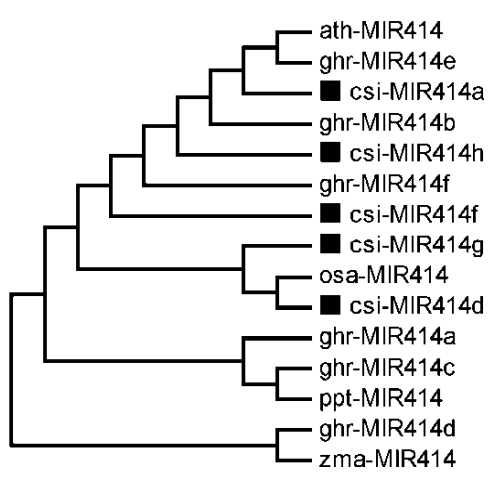

(a)

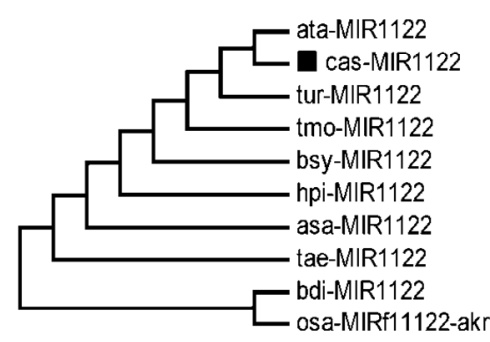

(b)

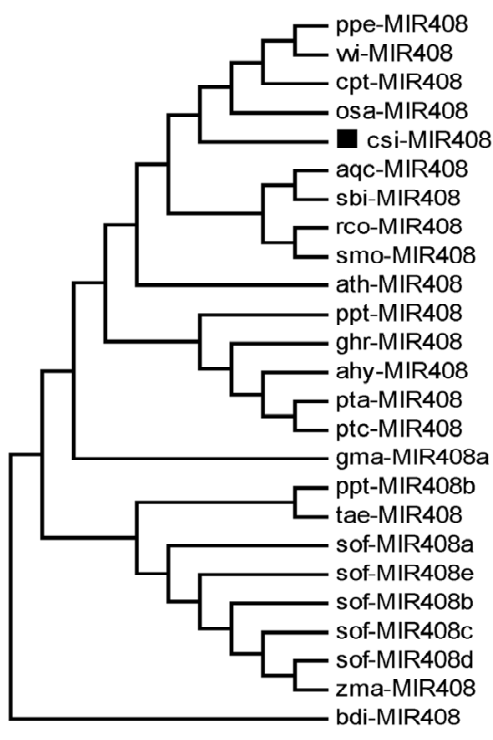

(c)

Figure 4. Phylogenetic relationships among the miRNA family members of (a) miRNA414 (b) miRNA1122 (c) miRNA408.

genes encoding different enzymes and $14 \%$ of genes encoding transporters as well as $48 \%$ of genes encoding various proteins of physiological and metabolic processes (Table 2). The miRNA family 'miR414' showed the highest 30 numbers of independent target genes followed by 'miR 408' family with 2 numbers of target genes. The rest miRNA families were with single target genes in Arabidopsis (Table 2). The 'miR1171' and 'miR1122' miRNA family members did not bind to any target sequences within our filtration criteria.

\section{Discussion}

With the availability of sequence resources in public databases, computer based miRNA identification methods have been focused more and more in the recent years due to its advantages of low cost and high efficiency. Sequence and structure homologies are the main theory behind the computer-based approach for miRNAs prediction. At present, four kinds of databases namely genome, GSS, EST and nucleotide are mainly used for plant miRNA mining. Considering the unavailability of genome and genomic survey sequences of tea, both EST and nucleotide databases were mined for miRNA identification. The number and sorts of miRNAs predicted in tea supported the fact that software-based approach is feasible and effective $[3,10,14]$.

The idenfied new miRNAs were belonged to 9 families where miR414 family has 5 members and the rests have single member in each. This familial distribution of miRNAs was also observed in Arabidopsis, rice and maize 
Table 2. Potential target genes of the identified miRNA families.

\begin{tabular}{|c|c|c|c|c|c|}
\hline $\begin{array}{c}\text { MiRNAs } \\
\text { Family }\end{array}$ & $\begin{array}{c}\text { Target } \\
\text { sites }\end{array}$ & Targeted proteins & Targets involved in & $\mathrm{EV}^{*}$ & Gene IDs \\
\hline \multirow[t]{2}{*}{$\operatorname{miR} 408$} & 1 & Cyclin-dependent protein kinase & regulation of cell cycle & 3.0 & TC284764 \\
\hline & 1 & Copper ion binding protein & metal ion transport & 3.0 & TC300377 \\
\hline \multirow[t]{31}{*}{$\operatorname{miR} 414$} & 1 & RAN GTPase activating protein 2 & cytokinesis & 1.0 & TC283936 \\
\hline & 1 & $50 \mathrm{~S}$ ribosomal protein $\mathrm{L} 21$ & translation process & 1.0 & TC290468 \\
\hline & 1 & 26S proteasome AAA-ATPase subunit RPT5a & proteasomal protein catabolic process & 1.0 & TC304323 \\
\hline & 1 & SEC12p-like transporter & ER to golgi vesicle mediated transpor & 1.5 & NP225634 \\
\hline & 1 & Nucleotide binding protein & nucleotide binding & 2.0 & TC280880 \\
\hline & 1 & MYB transcription factor & regulation of circadian rythm & 2.0 & TC283178 \\
\hline & 1 & Aldose 1-epimerase & carbohydrate metabolic process & 0.5 & TC285263 \\
\hline & 1 & Phosphatase $2 \mathrm{C}$-like protein & protein amino acid dephosphorylation & 0.5 & TC285483 \\
\hline & 1 & Phosphatidylinositol phosphatase & inositol or phosphatidylinositol activity & 0.5 & TC312518 \\
\hline & 1 & Starch branching enzyme class II & starch metabolic process & 1.0 & AA586097 \\
\hline & 1 & Reproductive meristem protein 1 & regulation of transcription & 1.0 & TC284340 \\
\hline & 1 & Calcium ion binding protein & calcium ion binding & 1.0 & TC299015 \\
\hline & 1 & Emb protein & RNA processing & 1.0 & TC294192 \\
\hline & 1 & Zinc ion binding protein & regulation of transcription & 1.0 & TC299076 \\
\hline & 1 & Calmodulin-4 & calcium ion binding & 1.5 & TC294389 \\
\hline & 1 & Translation initiation factor 3 subunit 8 & translation initiation & 0 & TC280751 \\
\hline & 1 & SMC3 protein & chromosome segregation process & 0 & TC281006 \\
\hline & 1 & MLO-like protein 3 & cell death & 0 & TC293173 \\
\hline & 1 & Ubiquitin conjugating enzyme & proteolysis & 0 & TC299050 \\
\hline & 1 & Ubiquitin conjugating enzyme & ubiquitin dependant protein catabolic process & 0.5 & CA781750 \\
\hline & 1 & Zinc finger protein & regulation of transcription & 0.5 & NP030706 \\
\hline & 1 & Plastid protein & protein targetting to chloroplast & 0.5 & TC290688 \\
\hline & 1 & Ubiquitin thiolesterase & ubiquitin dependant protein catabolic process & $\begin{array}{l}1.5 \\
1.5\end{array}$ & $\begin{array}{l}\text { TC280710, } \\
\text { TC298096 }\end{array}$ \\
\hline & & & & 1.5 & TC305774 \\
\hline & 1 & Methionyl-tRNA synthetase & methionyl-tRNA aminoacylation & 1.5 & TC282196 \\
\hline & 1 & Metal ion binding protein & metal ion binding & 1.5 & TC305801 \\
\hline & 1 & $\mathrm{Sfc} 4$ protein & xylem or phloem pattern formation & 2.0 & TC280894 \\
\hline & 1 & Transcription factor & regulation of transcription & $\begin{array}{l}2.0 \\
2.0\end{array}$ & $\begin{array}{l}\text { TC293875, } \\
\text { TC294793 }\end{array}$ \\
\hline & 1 & Synaptosomal-associated protein SNAP25 & vesicle mediated transport & 2.5 & TC293303 \\
\hline & 1 & ATP binding & protein amino acid phosphorylation & 2.5 & TC299397 \\
\hline & 1 & ADP-ribosylation factor-like protein & intracellular protein transport & 3.0 & TC289959 \\
\hline \multirow[t]{2}{*}{ miRf10132 } & 1 & Histone $\mathrm{H} 2 \mathrm{~B}$ like protein & nucleosome assembly & $\begin{array}{l}1.5 \\
1.5\end{array}$ & $\begin{array}{l}\text { TC297551, } \\
\text { TC313314 }\end{array}$ \\
\hline & & & & $\begin{array}{c}2.5 \\
1.5\end{array}$ & $\begin{array}{l}\text { TC313977, } \\
\text { TC294144 }\end{array}$ \\
\hline $\operatorname{miR} 2910$ & 1 & Extracellular matrix structural constituent & matrix organisation & 0 & TC310823, \\
\hline $\operatorname{miR} 2914$ & 1 & Glutamate semialdehyde dehydrogenase & glutamate metabolism & 2.0 & TC287905 \\
\hline $\operatorname{miRf10185}$ & 1 & Carboxylic ester hydrolase & hydrolase activity & $\begin{array}{l}3.0 \\
3.0\end{array}$ & $\begin{array}{l}\text { TC298946, } \\
\text { TC308821 }\end{array}$ \\
\hline $\operatorname{miR} 11590$ & 1 & FRIGIDA protein & regulation of flower development & 2.0 & TC309547 \\
\hline
\end{tabular}

$* \mathrm{EV}=$ Expectation value 


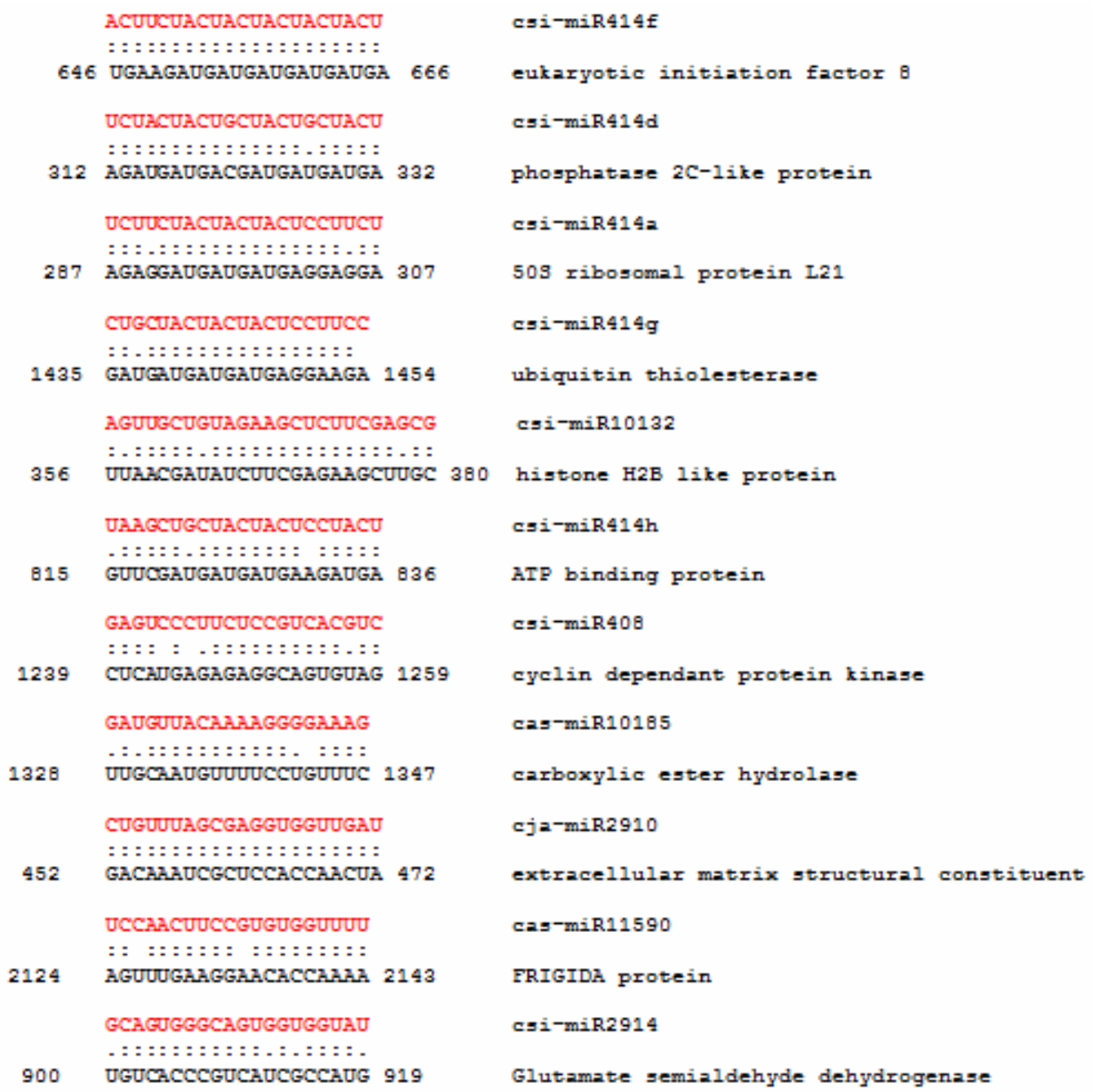

Figure 5. Predicted miRNA targets (black in colour) and their complementary sites with miRNAs (red in colour).

[29]. This may be an indicative of dominant nature of miR414 family in miRNA-mediated gene regulation in tea. The miRNAs were found diverse in nature such as location of mature miRNA sequences and length of precursor sequences. The average length of precursor sequence was 248 nucleotides; however a majority of them (62\%) have 65-200 nucleotides. This finding is similar to other plants where the length of precursors varied in contrast to consistent miRNA length of animal miRNAs (70-80 nt) [30,31]. In tea miRNAs, diversity was also observed within the members of same family which was also found in maize [1]. The identified precursor miRNAs were fold into hairpin secondary structures using minimum free energies, with an average -72.69 $\mathrm{kcal} / \mathrm{mol}$ which was lower than the values of Arabidopsis thaliana precursor miRNAs and much lower than the folding free energies of tRNA $(-27.5 \mathrm{kcal} / \mathrm{mol})$ and rRNA $(-33 \mathrm{kcal} / \mathrm{mol})[32]$.
Out of 13 newly identified miRNAs, ten were from ESTs. There are several reports on miRNA identification from ESTs in various plant species $[1,33]$. The source sequences of miRNAs show a link between miRNAs and their tissues, organs, developmental stages or expression to which it belongs. On that basis, it was recognized that csi-miR414f, cas-miRf10185 and cas-miR11590-akr might be expressed in root and the rests were in leaf tissues. Moreover, csi-miR1171 and csi-miR414d were found in leaf tissue under the stress of winter dormancy and pest infestation, respectively. Three miRNAs namely casmiR1122, cja-miR2910 and csi-miR2914 were identified from the full length nucleotides of RNA polymerase second largest subunit (intron 23) and 18S ribosomal subunit, respectively. Plant miRNAs are highly conserved among distantly related plant species, both in terms of primary and mature miRNAs [4]. This finding is also supported by our results, the identified miRNA was 
found conserved in diverse plant species from monocotyledonous to dicotyledonous plants. These results suggested that different miRNAs might have evolved at different rates not only within the same plant species, but also in different ones.

The miRNA target gene identification is an important step for understanding the role of miRNAs in gene regulatory networks. Our prediction of target genes for the tea miRNAs revealed that more than one gene was regulated by individual miRNA. This result was similar to the recent findings in other plant species $[1,10]$ which suggested that miRNA research should be focused on networks rather than individual connections between miRNA and strongly predicted targets. MiRNAs may directly target transcription factors which affect plant growth and development, and also specific genes which control metabolism [4]. In this study, we identified a total of 37 potential targets for the 7 identified miRNA families in tea. The identified target genes appeared to be associated with diverse biological functions. There were genes encoding transcription factors such as MYB, translation intiation factor such as TIF3, important proteosome degrading pathway enzyme such as ubiquitin conjugating enzyme, different ion transporters such as copper ion binding protein, carbohydrate metabolism related enzyme such as aldose 1-epimerase, glutamate metabolism related enzymes such Glutamate semialdehyde dehydrogenase, important protein for nucleosome assembly such as histone as well as ribosomal proteins. In an earlier report, it was found as $20 \%$ transcription factors and $53 \%$ proteins related to diverse physiological processes, however their investigation was limited to only four miRNAs [34]. Overall, these findings made us clear that tea miRNAs targeted both trancription factors as well as specific genes.

This findings of miRNAs in tea will pave the way for understanding the function and processing of tea small RNAs in future. Moreover, it shows a path for the prediction and analysis of miRNAs to those species whose genomes are not available through bioinformatics tools.

\section{REFERENCES}

[1] B. Zhang, X. Pan and A. T. Anderson, "Identification of 188 Conserved Maize microRNAs and Their Targets", FEBS Letters, Vol. 580, No. 15, June 2006, pp. 3753-3762.

[2] X. Zhou, J. Ruan, G. Wang and W. Zhang, "Characterization and Identification of MicroRNA Core Promoters in Four Model Species," PLoS Computational Biology, Vol. 3, No. 3, January 2007, p. e37.

[3] J. Singh and J. Nagaraju, "In silico Prediction and Characterization of microRNAs from Red Flour Beetle (Tribolium castaneum)," Insect Molecular Biology, Vol. 17, No. 4, August 2008, pp. 427-436.
[4] B. Zhang, X. Pan, G. P. Cobb and T. A. Anderson, "Plant microRNA: A Small Regulatory Molecule with Big Impact," Developmental Biology, Vol. 289, No. 1, January 2006, pp. 3-16.

[5] C. A. Mallory and H. Vaucheret, "Functions of microRNAs and Related Small RNAs in Plants," Nature Genetics, Vol. 38, May 2006, pp. S31-S36.

[6] R. Sunkar, V. Chinnusamy, J. Zhu and K. J. Zhu, "Small RNAs as Big Players in Plant Abiotic Stress Responses and Nutrient Deprivation," Trends in Plant Science, Vol. 12, No. 7, July 2007, pp. 301-309.

[7] B. Zhang, X. Pan, C. H. Cannon, G. P. Cobb and A. T. Anderson, "Conservation and Divergence of Plant microRNA Genes," Plant Journal, Vol. 46, No. 2, April 2006, pp. 243-259.

[8] A. N. Eckardt, "MicroRNAs Regulate Auxin Homeostasis and Plant Development," The Plant Cell, Vol. 17, May 2005, pp. 1335-1338.

[9] S. H. Guo, Q. Xie, F. J. Fei and H. N. Chua, "MicroRNA Directs mRNA Cleavage of the Transcription Factor NAC1 to Downregulate Auxin Signals for Arabidopsis Lateral Root Development," The Plant Cell, Vol. 17, May 2005, pp. 1376-1386.

[10] M. W. Jones-Rhoades and P. D. Bartel, "Computtional Identification of Plant microRNAs and Their Targets, Including a Stress-Induced miRNA," Molecular Cell, Vol. 14, June 2004, pp. 787-799.

[11] R. Sunkar and J. K. Zhu, "Novel and Stress-Regulated microRNAs and Other Small RNAs from Arabidopsis", The Plant Cell, Vol. 16, No. 8, August 2004, pp. 20012019.

[12] Y. Lu, Q. Gan, X. Chi and S. Qin, "Roles of microRNA in Plant Defense and Virus Offense Interaction," Plant Cell Reports, Vol. 27, No. 10, October 2008, pp. 15711579.

[13] R. Alba, Z. Fei, P. Payton, Y. Liu, L. S. Moore, P. Debbie, J. Cohn and M. D'Ascenzo, "ESTs, cDNA Microarrays, and Gene Expression Profiling: Tools for Dissecting Plant Physiology and Development," The Plant Journal, Vol. 39, No. 5, September 2004, pp. 697-714.

[14] B. H. Zhang, X. P. Pan, Q. L. Wang, G. P. Cobb and T. A. Anderson, "Identification and Characterization of New Plant microRNAs Using EST Analysis," Cell Research, Vol. 15, No. 5, May 2005, pp. 336-360.

[15] Q. Guo, A. Xiang, Q. Yang and Z. Y. Yang, "Bioinformatic Identification of microRNAs and Their Target Genes from Solanum tuberosum Expressed Sequence Tags," Chinese Science Bulletin, Vol. 52, No. 17, September 2007, pp. 2380-2389.

[16] M. N. Nasaruddin, K. Harikrishna, Y. R. Othman, S. L. Hoon and A. J. Harikrishna, "Computational Prediction of microRNAs from Oil Palm (Elaeis guineensis Jacq.) Expressed Sequence Tags," Asian Pacific Journal of Molecular Biology and Biotechnology, Vol. 15, No. 3, October 2007, pp. 107-113.

[17] W. Jin, N. Li, B. Zhang, F. Wu, W. Li, A. Guo and Z. Deng, "Identification and Verification of microRNA in 
Wheat (Triticum aestivum)," Journal of Plant Research, Vol. 121, No. 3, March 2008, pp. 351-355.

[18] B. Billoud, D. R. Paepe, D. Baulcombe and M. Boccara, "Identification of New Small Non-Coding RNAs from Tobacco and Arabidopsis," Biochimie, Vol. 87, No. 9, September 2005, pp. 905-910.

[19] R. Sunkar and G. Jagadeeswaran, "In silico Identification of Conserved microRNAs in Large Number of Diverse Plant Species,” BMC Plant Biology, Vol. 8, No. 37, 2008, p. 13.

[20] S. Griffiths-Jones, H. K. Saini, D. S. Van and A. J. Enright, "MiRBase: Tools for microRNA Genomics," Nucleic Acids Research, Vol. 36, November 2008, pp. D154D158.

[21] N. Rajewsky and D. N. Socci, "Computational Identification of microRNA Targets," Developmental Biology, Vol. 267, No. 2, March 2004, pp. 529-535.

[22] L. Zhang, M. J. Chia, S. Kumari, C. J. Stein, Z. Liu, A. Narechania, A. C. Maher, K. Guill, D. M. McMullen and D. Ware, "A Genome-Wide Characterization of MicroRNA Genes in Maize," PLoS Genetics, Vol. 5, No. 11, November 2009, pp. 5e1000716.

[23] S. F. Altschul, T. L. Madden, A. A. Schäffer, J. Zhang, Z. Zhang, W. Miller and D. J. Lipman, "Gapped BLAST and PSI-BLAST: A New Generation of Protein Database Search Programs," Nucleic Acids Research, Vol. 25, No. 17, September 1997, pp. 3389-3402.

[24] M. Zuker, "Mfold Web Server for Nucleic Acid Folding and Hybridization Prediction," Nucleic Acids Research, Vol. 31, No. 13, July 2003, pp. 3406-3415.

[25] J. D. Thompson, D. G. Higgins and T. J. Gibson, "CLUSTAL W: Improving the Sensitivity of Progressive Multiple Sequence Alignments through Sequence Weighting, Position Specific Gap Penalties and Weight Matrix Choice," Nucleic Acids Research, Vol. 22, No. 22, November 1994, pp. 4673-4680.

[26] K. Tamura and M. Nei, "Estimation of the Number of

Abbreviations used: BLAST, basic local alignment search tool; dbEST, database of expressed sequence tags; ESTs, expressed sequence tags; mRNA, messenger RNA;
Nucleotide Substitutions in the Control Region of Mitochondrial DNA in Humans and Chimpanzees," Molecular Biology and Evolution, Vol. 10, No. 3, May 1993, pp. 512-526.

[27] K. Tamura, J. Dudley, M. Nei and S. Kumar, "MEGA4: Molecular Evolutionary Genetics Analysis (MEGA) software version 4.0.," Molecular Biology and Evolution, Vol. 24, No. 8, August 2007, pp. 1596-1599.

[28] S. Griffiths-Jones, R. J. Grocock, D. S. Van, A. Bateman and A. J. Enright, "miRBase: microRNA Sequences, Targets and Gene Nomenclature," Nucleic Acids Research, Vol. 34, November 2006, pp. D140-D144.

[29] E. Bonnet, J. Wuyts, P. Rouze and Y. V. de Peer, "Evidence that microRNA Precursors, unlike Other Non-Coding RNAs, Have Lower Folding Free Energies than Random Sequences," Bioinformatics, Vol. 20, No. 17, November 2004, pp. 2911-2917.

[30] P. D. Bartel, "MicroRNAs: Genomics, Biogenesis, Mechanism and Function," Cell, Vol. 116, No. 2, January 2004, pp. 281-297.

[31] V. Ambros, "The Functions of Animal microRNAs," Nature, Vol. 431, September 2004, pp. 350-355.

[32] M. Y. Khan-Barozai, M. Irfan, R. Yousaf, I. Ali, U. Qaisar, A. Maqbool, M. Zahoor, B. Rashid, T. Hussnain and S. Riazuddin, "Identification of microRNA in Cotton," Plant Physiology and Biochemistry, Vol. 46, No. 8, August 2008, pp. 739-751.

[33] C. X. Qiu, F. L. Xie, Y. Y. Zhu, K. Guo, S. Q. Huang, L. Nie and Z. M. Yang, "Computational Identification of microRNAs and Their Targets in Gossypium hirsutum Expressed Sequence Tags," Gene, Vol. 395, No. 1, June 2007, pp. 49-61.

[34] G. R. Prabu and A. K. A. Mandal, "Computational Identification of miRNAs and Their Target Genes from Expressed Sequence Tags of Tea (Camellia sinensis)," Genomics Proteomics and Bioinformatics, Vol. 8, No. 2, June 2010, pp. 113-121.
miRNA, microRNA; mfe, minimum free energy; nt, nucleotide; NCBI, national center for biotechnological information 